\title{
Observation of surface-initiated melting
}

\author{
Joost W. M. Frenken, Peter M. J. Marée, and J. Friso van der Veen \\ FOM-Institute for Atomic and Molecular Physics, Kruislaan 407, NL-1098 SJ Amsterdam, The Netherlands
}

(Received 20 June 1986)

\begin{abstract}
Ion-shadowing and blocking measurements reveal a reversible order-disorder transition at the (110) surface of a lead crystal well below its melting point $T_{m}$. The transition starts with partial disordering of the surface region at approximately $0.75 T_{m}$. Closer to $T_{m}$ a completely disordered film builds up with a thickness that increases rapidly as the temperature approaches $T_{m}$. Electron diffraction patterns show the loss of two-dimensional order of the $\mathrm{Pb}(110)$ surface, at a temperature where the surface is nearly completely disordered. A detailed analysis of the surface cleanliness provides evidence against the possible role of surface impurities in the observed effects.
\end{abstract}

\section{INTRODUCTION}

Although melting is one of the most common phase transitions, a generally accepted theory of the solid-liquid transition on an atomic level is still lacking. What is known is largely based on thermodynamics. Melting is a first-order phase transition, characterized by a discontinuous change in entropy. At the melting point $T_{m}$, the Gibbs free energy per mole of the bulk liquid $G_{l}$ is equal to that of the solid $G_{s}$, and the two phases coexist. One of the peculiar characteristics of melting is that under normal conditions superheating of solids above $T_{m}$ is not observed, whereas undercooling of liquids is. Apparently, there is no energetic barrier for nucleation of melting, while such a barrier does exist for solidification. A possible cause for this asymmetry could be the occurrence of premelting phenomena at temperatures below $T_{m}$, which would pave the way for melting. For various materials premonitory symptoms of melting have been observed in macroscopic properties such as heat conductivity, electrical conductivity, self-diffusion coefficient, etc. ${ }^{1,2}$

In the past, several theoretical attempts to provide a microscopic picture of melting have invoked some thermally induced instability of the crystal lattice at $T_{m}$. Such a lattice instability would be the result of strong lattice vibrations, ${ }^{1-4}$ the vanishing of shear moduli, ${ }^{5}$ or the spontaneous generation of a high concentration of crystal imperfections (vacancies, ${ }^{6}$ interstitials, ${ }^{7}$ dislocations ${ }^{8}$ ). Experiments however, have revealed no sign of such a mechanism. Neither the softening of a bulk phonon, nor the vanishing of the bulk rigidity modulus, nor the massive production of bulk defects in crystals close to $T_{m}$ have been observed. ${ }^{2,3,9}$

Theories ${ }^{10-14}$ and computer experiments ${ }^{15-20}$ which explicitly account for the limited size of a real crystal attribute a key role to the surface in the melting transition. The fact that surface atoms have a reduced number of nearest neighbors could make the temperature at which the surface becomes unstable lower than that for the bulk. $^{21}$ If the surface were to actually melt before the bulk, it would serve as a vast two-dimensional nucleus for bulk melting at $T_{m}$, at which temperature the solid dis- solves in its own surface melt. ${ }^{22}$ This mechanism would explain the common absence of superheating (only if the influence of crystal surfaces is suppressed, superheating becomes possible ${ }^{23-27}$ ). The often satisfied inequality ${ }^{3}$

$$
\gamma_{s v}>\gamma_{s l}+\gamma_{l v}
$$

between the interfacial free energies $\gamma$ per unit area of, respectively, the solid-vapor, the solid-liquid, and the liquid-vapor interfaces, is in support of this idea. It suggests that for most solids it is indeed thermodynamically favorable to be covered by a liquid film just below $T_{m}$.

Recently, Lipowsky and Speth ${ }^{10,11}$ put surface melting in a somewhat broader perspective. Using Landau theory they have shown that, in general, semi-infinite systems undergoing a first-order phase transition in the bulk may exhibit critical behavior at the surface: surface quantities may behave continuously although bulk quantities change abruptly.

The aim of this paper is to give a detailed account of the first direct experimental observation of a reversible melting transition of the surface of a three-dimensional crystal. Temperature-dependent ion scattering measurements on an atomically clean $\mathrm{Pb}(110)$ surface reveal the presence of a disordered film on top of, and in equilibrium with, a well-ordered substrate. In Sec. II, experimental details are given concerning the preparation, surface cleanliness and temperature control of the $\mathrm{Pb}(110)$ specimens. The ion scattering measurements and their interpretation form the subject of Sec. III. In Sec. IV we present reflection high-energy electron diffraction (RHEED) observations of this surface order-disorder transition. In Sec. $\mathrm{V}$ the infrared emissivity of $\mathrm{Pb}(110)$ will be shown to indicate surface melting below $T_{m}$ as well. Finally, from the experimental information a model of surface melting is constructed, which is then discussed in the light of the existing literature.

\section{SAMPLE PREPARATION}

For this investigation the $\mathrm{Pb}(110)$ surface was selected for three reasons. Firstly, the low melting point of $\mathrm{Pb}$, $T_{m}=600.7 \mathrm{~K}$, is easily reached and facilitates accurate 
temperature control. Secondly, the vapor pressure of $\mathrm{Pb}$ at $T_{m}$ is only $\sim 7 \times 10^{-7} \mathrm{~Pa}^{28}$ This corresponds to an evaporation rate of $\sim 3$ monolayers per hour. Melting under ultrahigh vacuum (UHV) conditions is therefore expected to proceed very similarly to melting in equilibrium with $\mathrm{Pb}$ vapor (triple point). Finally, the most pronounced surface premelting effect may be expected on an open surface, ${ }^{12-14}$ such as the (110) surface of $\mathrm{Pb}$ (fcc structure).

$\mathrm{Pb}$ specimens, with dimensions of $12 \times 12 \times 5 \mathrm{~mm}^{3}$, were spark-cut from a single-crystal $\mathrm{Pb}$ bar of $99.99 \%$ purity (Metal Crystals Ltd., Cambridge, U.K.). An etchpolish mixture of $80 \%$ acetic acid and $20 \%$ hydrogen peroxide $^{29}$ was used to remove the damaged surface region and to obtain a smooth, shiny surface. Two grooves in the sides of the crystal were used to clamp it gently in a $\mathrm{Cu}$ or Mo container. The (110) surface was cleaned in situ by cycles of argon ion bombardment $\left(2 \times 10^{15}\right.$ ions $/ \mathrm{cm}^{2}$ of $700-\mathrm{eV}$ energy, at a temperature of $550 \mathrm{~K}$ ) and annealing ( $1 \mathrm{~h}$ at $590 \mathrm{~K}$ ), until no impurities were detected with Auger electron spectroscopy (AES) and the surface was well ordered, as seen with both low-energy electron diffraction (LEED) and ion channeling (low minimum yield). All measurements were performed in UHV (pressure below $1.5 \times 10^{-8} \mathrm{~Pa}$ ).

The preparation of an atomically clean surface is important, since some impurity elements lower the melting point appreciably. ${ }^{30}$ Table I lists the common meltingpoint lowering elements and their usual bulk concentrations for $\mathrm{Pb}$ of $99.99 \%$ purity. ${ }^{31}$ The expected surface concentrations, calculated from these bulk concentrations with use of surface segregation theory, ${ }^{32,33}$ are shown as well. The surface cleanliness of $\mathrm{Pb}(110)$ was checked experimentally with AES at different temperatures up to 594 K. Figure 1 shows a differentiated electron energy spectrum at $594 \mathrm{~K}$, measured with a cylindrical-mirror energy analyzer (CMA) and channeltron for electron counting, and corrected for the transmission function of the CMA. The primary-electron energy was $3 \mathrm{keV}$. Spec-

TABLE I. Common impurity elements and their nominal bulk concentrations $C_{b}$ in $99.99 \%$-pure $\mathrm{Pb}$ (Ref. 31 ). The expected surface concentrations $C_{s}$ were calculated using Refs. 32 and 33. The AES detection limits are given in monolayers with respect to the $\mathrm{Pb}(110)$ substrate. The last column shows the melting point depressions expected when amounts equal to these detection limits would be dissolved in one monolayer of $\mathrm{Pb}$ (Ref. 30).

\begin{tabular}{ccccc}
\hline \hline $\begin{array}{c}\text { Impurity } \\
\text { element }\end{array}$ & $\begin{array}{c}C_{b} \\
(\mathrm{ppm})\end{array}$ & $\begin{array}{c}C_{s} \\
(\mathrm{ppm})\end{array}$ & $\begin{array}{c}\text { AES } \\
\text { detection limit } \\
(\text { monolayer })\end{array}$ & $\begin{array}{c}\Delta T_{m} \\
(\mathbf{K})\end{array}$ \\
\hline $\mathrm{Bi}$ & 50 & 140 & 0.030 & -8.0 \\
$\mathrm{Ag}$ & 10 & 0.06 & 0.003 & -1.5 \\
$\mathrm{Cu}$ & 10 & 0.2 & 0.060 & $-1.0^{\mathrm{a}}$ \\
$\mathrm{Sb}$ & 10 & 3 & 0.005 & -2.2 \\
$\mathrm{Zn}$ & $<10$ & $<3$ & 0.003 & -1.7 \\
$\mathrm{Sn}$ & $<10$ & $<3$ & 0.006 & -1.0 \\
$\mathrm{As}$ & $<10$ & $<0.2$ & 0.012 & -6.3 \\
\hline \hline
\end{tabular}

${ }^{a}$ Maximum melting point depression, for eutectic at 0.02 at $\%$ $\mathrm{Cu}$.

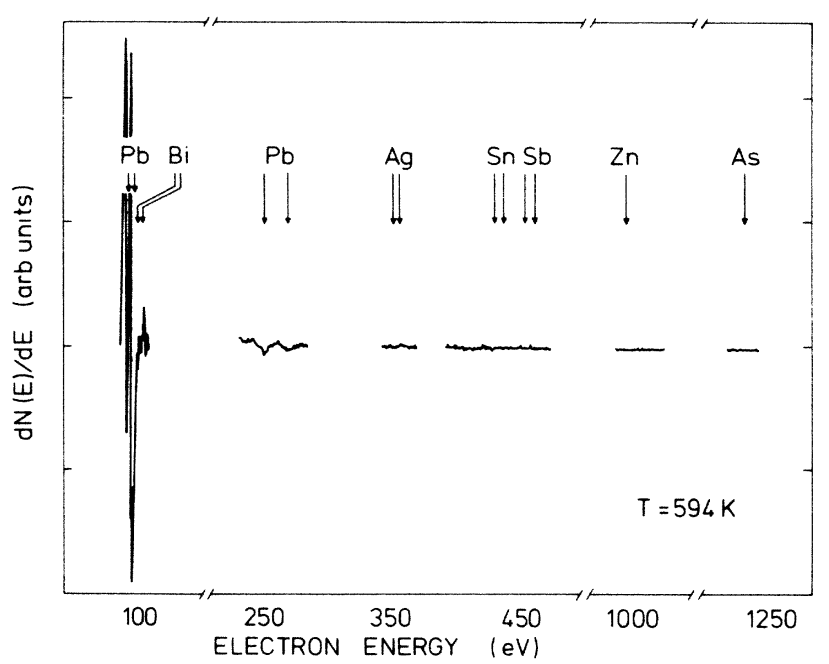

FIG. 1. AES spectrum from $\mathrm{Pb}(110)$ at a temperature of 594 K. The primary electron energy was $3 \mathrm{keV}$. The arrows indicate expected peak positions for the impurity elements in Table I.

tra obtained at lower temperatures are identical to the one in Fig. 1 to within statistical error. The energy ranges shown cover the Auger peak positions of the elements in Table I. No trace is visible of any other element than $\mathrm{Pb}$. The AES detection limits and the corresponding melting point depressions $\Delta T_{m}$ that these quantities would cause when dissolved per monolayer of $\mathrm{Pb}$, are also given in Table I. Note that these estimates of $\Delta T_{m}$ are upper limits for the impurity-induced surface melting-point depression in that the full amount equivalent to the AES detection limit is assumed to be present in the first layer, and not in any of the subsurface layers. Most impurities, however, are not expected to exhibit any enrichment at the $\mathrm{Pb}$ surface (Table I), because of the low surface energy of $\mathrm{Pb}^{32,33}$ Only for $\mathrm{Bi}$ is a surface-to-bulk concentration ratio of $\sim 2.7$ expected, which would still result in a negligibly small amount at the surface. The observed onsets of partial disordering and complete disordering of the surface, at 150 and $20 \mathrm{~K}$ below $T_{m}$, respectively (see Sec. III), cannot be explained by any of the entries in Table I. Also for other melting-point lowering elements, not listed in Table I (such as In), the AES detection limits correspond to melting point depressions much smaller than 10 K. Temperature cycles up to $T_{m}$ did not result in any accumulation of impurities at the surface. All measurements reported in this paper were reproducible in time and from sample to sample.

The surface temperature was continuously monitored by an infrared pyrometer which was calibrated against a Pt resistance thermometer, embedded in the sample container. The temperature scale was fixed by the bulk melting point of $\mathrm{Pb}$, which was measured in situ. The accuracy of this calibration is within $\pm 0.1 \mathrm{~K}$. The crystal was heated by electron bombardment or radiative heating of the back of its container. In this configuration the front of our samples is estimated to be $0.01 \mathrm{~K}$ colder than the back, at temperatures close to $T_{m}$. The temperature stability was better than $\pm 0.3 \mathrm{~K}$ throughout the whole temperature range, and better than $\pm 0.1 \mathrm{~K}$ close to $T_{m}$. 


\section{SHADOWING AND BLOCKING}

\section{A. Method}

The shadowing and blocking technique ${ }^{34}$ is schematically represented in Fig. 2. A parallel proton beam of 97.5-keV energy is aligned with the [101] crystal axis of the specimen. Protons impinging close to a first-layer atom are deflected away from their original direction. In case of an ideal static lattice the resulting shadow cones behind the outermost atoms completely cover all second, third and deeper-layer atoms [Fig. 2(a)]. A particle detector would in that case only collect protons backscattered from surface atoms. Thermal vibrations blur the shadow cones so that also atoms in deeper layers contribute to backscattering. An electrostatic energy analyzer is used to detect protons emerging from the crystal parallel to the [011] axis. Blocking of backscattered protons along this direction further reduces the backscattering yield from subsurface atoms. A backscattering energy spectrum [Fig. 2(a)] consists of a surface peak (SP) from the exposed surface layers and a low "minimum yield" from the small nonshadowed, nonblocked fraction of deeper layers. The latter contribution appears at lower energies due to the electronic stopping of protons in the material. ${ }^{35}$ The stopping makes the energy spectrum an inverted depth spectrum (see Sec. III C).

If the crystal is covered by a disordered (molten) film, coherent shadow cones and blocking cones are only formed below the melt-crystal interface [Fig. 2(b)]. All atoms in the film are fully visible to both beam and detector, i.e., they contribute fully to the surface backscattering yield, thereby increasing area and width of the SP. Such changes in SP shape are monitored on a monolayer scale, owing to the high-energy resolution of the electrostatic energy analyzer. The measured energy resolution function is approximately Gaussian with a full width at half maximum (FWHM) of $\delta E=4 \times 10^{-3} E$, where $E$ is the ion energy. Combining the resolution of $\delta E=390 \mathrm{eV}$ for
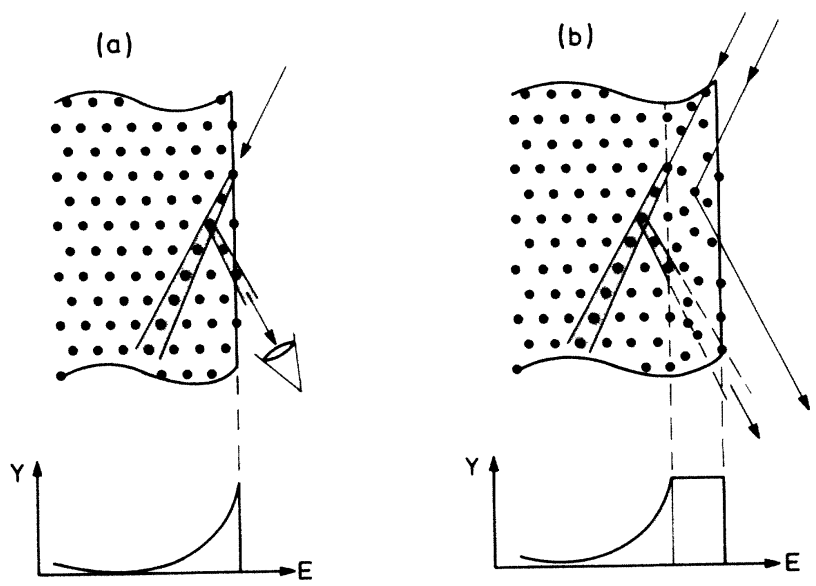

FIG. 2. Schematic representation of energy spectra for (a) a well-ordered crystal surface, and (b) a crystal covered by a disordered surface layer. Ion beam and detector are aligned with the [101] and [011] directions in the (1T1) crystal plane. Shadowing and blocking effects are indicated.
$E=97.5 \mathrm{keV}$ with a stopping power of $13.7 \mathrm{eV}$ per $\AA$ path length, ${ }^{35}$ i.e., $55 \mathrm{eV}$ per $\AA$ depth, we obtain a depth resolution of $7.1 \AA$ or 4.1 monolayers (see Sec. III C).

Independently from the energy to depth conversion the area under the SP can be calibrated (within an accuracy of $\pm 5 \%$ ) to give the number of $\mathrm{Pb}$ monolayers visible to both proton beam and detector. One calibration procedure makes use of the known scattering yield from a backscattering standard and the fraction of protons neutralized at the $\mathrm{Pb}$ surface, which was measured to be $16.0 \pm 1.0 \%$ for $97.5-\mathrm{keV}$ protons. ${ }^{36}$ In addition, an internal calibration is obtained from the backscattering yield from a molten lead sample, the so-called "random height," and the (random) stopping power. ${ }^{35}$ The two calibrations agree well within the experimental error margins.

The toroidal shape of the energy analyzer allows for the simultaneous accumulation of a large set of energy spectra in a $20^{\circ}$ angular range. All energy spectra shown in this paper correspond to a $1.8^{\circ}$ window around the [011] crystal direction.

\section{B. Results}

Figure 3 shows a selection of measured energy (depth) spectra, calibrated with respect to the random height (all atoms fully visible). The spectrum at $295 \mathrm{~K}$ is indicative of a well-ordered surface. Its SP area corresponds to only 2.5 visible monolayers while deeper layers are almost completely shadowed or blocked. Up to $450 \mathrm{~K}$ the SP increases slowly in area and width. Above this temperature the SP grows at an increased rate, reaching the random height at $\sim 599 \mathrm{~K}$. From this it is inferred that a depth region of about twice the FWHM depth resolution, i.e., 8 monolayers, is fully visible at that temperature. ${ }^{37}$ At still higher temperatures, the SP rapidly increases further in

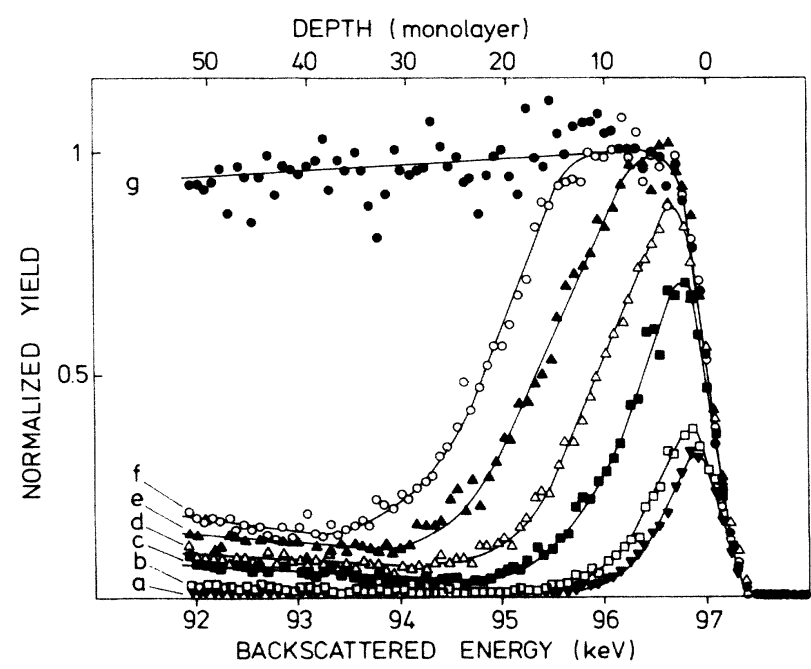

FIG. 3. Experimental energy spectra obtained with $97.5-\mathrm{keV}$ protons in the scattering geometry of Fig. 2, and calibrated with respect to the random height: (a) $295 \mathrm{~K}$; (b) $452 \mathrm{~K}$; (c) $581 \mathrm{~K}$; (d) $597 \mathrm{~K}$; (e) $599.7 \mathrm{~K}$; (f) $600.5 \mathrm{~K}$; and (g) $600.8 \mathrm{~K}$; $T_{m}=600.7$ K. Solid curves serve to guide the eye. 
width. Here the spectra strongly resemble the schematic SP for surface melting [Fig. 2(b)]. If the sample is deliberately melted [Fig. 3, spectrum $(g)$ ], the energy spectrum shows absolutely no shadowing or blocking, as is expected for a bulk liquid. ${ }^{38}$ The energy spectra in Fig. 3 do not change with beam current, measuring time or total beam dose.

The temperature dependence of the number of visible $\mathrm{Pb}$ layers calculated from the SP area is shown in Fig. 4. This dependence was found to be fully reversible (as long as $\left.T<T_{m}\right)$ with no indication of hysteresis on the time scale of each measurement $(\sim 30 \mathrm{sec})$.

Results consistent with those in Fig. 3 and Fig. 4 were obtained using a $175-\mathrm{keV}$ beam of $\mathrm{He}^{+}$ions. Better temperature control $( \pm 0.05 \mathrm{~K})$ enabled us in this case to measure "random spectra" (i.e., more than $\sim 40$ fully visible layers) at a temperature within $0.05 \mathrm{~K}$ from $T_{m}$. Again, full reversibility was found: upon cooling a sharp SP returned immediately, with a low minimum yield.

In Fig. 5 energy spectra are shown over an angular range of $20^{\circ}$ around the [011] bulk axis, at a temperature of $600.5 \mathrm{~K}$. Apart from a smooth dependence on the scattering angle, resulting from the Rutherford backscattering cross section, no angular variations, such as blocking or flux peaking effects, are present in the height of the SP, whereas at lower energies a distinct blocking minimum is seen along the [011] direction. This indicates that well-defined crystal axes or crystal planes are absent in the depth region corresponding to the SP width at this temperature, while the crystallinity of the bulk is apparent.

\section{Discussion}

In Fig. 4 three different temperature regimes can be identified, which will be analyzed with the aid of comput-

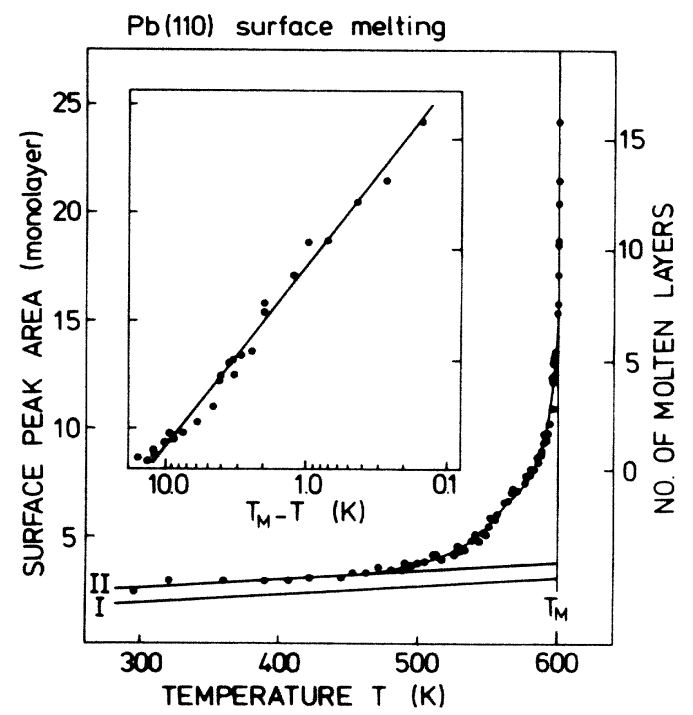

FIG. 4. Calibrated surface-peak area as a function of temperature. The vertical line indicates the bulk melting point $T_{m}$. The inset is an expanded view of the highest $20-\mathrm{K}$ interval, on a logarithmic temperature scale (see Sec. IV D). Curves I and II are discussed in the text. The right-hand vertical axis (Refs. 45 and 46) is obtained from the analysis in Sec. III C.

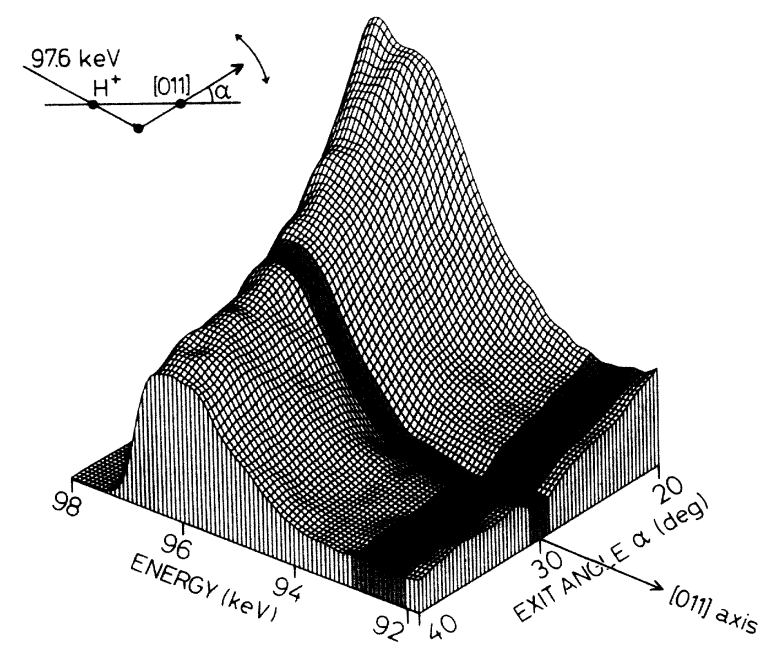

FIG. 5. Energy spectra measured at $600.5 \mathrm{~K}$ in a $20^{\circ}$ angular range around the [011] bulk axis. The shaded portions denote the $1.8^{\circ}$ angular window which is used to produce the energy spectra in Fig. 3, and the energy window, from 92 to $93 \mathrm{keV}$, which is used to produce the bulk blocking pattern in Fig. 6(a). The data have been smoothed for presentational purposes. The inset shows the scattering geometry (see also Fig. 2).

er simulations of the backscattering experiment. We will first show that the modest increase up to $\sim 450 \mathrm{~K}$ corresponds to a well-ordered surface. Then the steep part at high temperatures, from $\sim 580 \mathrm{~K}$ up to $T_{m}$, will be explained by the growth of a disordered surface layer. At intermediate temperatures, between 450 and $580 \mathrm{~K}$, there is evidence for a gradual disordering of the surface region.

The experiment was simulated with a Monte Carlo computer code. ${ }^{39}$ For such a simulation $\sim 10^{5}$ ion trajectories are constructed through a slab of 40 atomic layers, using the Molière scattering potential. The backscattering yield of each layer along the trajectories is efficiently acquired with the nuclear encounter probability method. ${ }^{40}$ Lattice vibrations are modelled by Gaussian probability densities of the atoms around their average positions (quasiharmonic approximation ${ }^{41}$ ). The bulk thermalvibration amplitude $\sigma_{\text {bulk }}$ (defined as the one-dimensional rms thermal displacement of bulk $\mathrm{Pb}$ atoms) was taken from Ref. 41 . It increases almost linearly from $0.18 \AA$ at room temperature to $0.28 \AA$ just below $T_{m}$. This causes the SP area calculated for a bulklike solid surface to follow curve $I$ in Fig. 4. Curve II is obtained by also accounting for a $50 \%$ enhanced vibration amplitude of the outermost atomic layer and relaxations of the topmost four interlayer distances by $\Delta d_{12} / d=-15.9 \%, \Delta d_{23} / d$ $=+7.9 \%, \Delta d_{34} / d=-6.8 \%$, and $\Delta d_{45} / d=+0.7 \%(d$ being the bulk interlayer distance of $1.75 \AA$ ), as found in a combined theoretical and experimental study of $\mathrm{Pb}(110)$ at room temperature. ${ }^{36}$ Curve II matches the first temperature regime in Fig. 4. From this we conclude that the surface is well ordered up to $450 \mathrm{~K} .{ }^{42}$

Above $450 \mathrm{~K}$ the measured SP areas rise above those in curve II. The difference amounts to as much as 20 extra visible $\mathrm{Pb}$ layers at $600.5 \mathrm{~K}$. These large $\mathrm{SP}$ areas cannot be simulated by simply assuming an ordered surface and a 
high bulk vibration amplitude. Even for unrealistically large $\sigma_{\text {bulk }}$ values (such as $\sigma_{\text {bulk }} \geq 1 \AA$ ) the simulated energy spectra do not fit any of the observed spectra. Moreover, from angular distributions of the scattering yield just below the SP in the energy spectra, it was deduced that $\sigma_{\text {bulk }}$ does not become excessively large. Figure 6(a) displays the angular distribution of the scattering yield between 92 and $93 \mathrm{keV}$, i.e., from a depth region between 41 and 51 monolayers, at a temperature of $600.5 \mathrm{~K}$ (see Fig. 5). The width of the [011] blocking dip is directly related to the value of $\sigma_{\text {bulk }}$ at this temperature. ${ }^{44}$ In Fig. $6(\mathrm{~b})$ the measured widths (FWHM) have been plotted versus temperature. The solid line in Fig. 6(b) is the result of Monte Carlo simulations using the $\sigma_{\text {bulk }}$ values from Ref. 41 mentioned before. The agreement between measured and calculated widths excludes a high $\sigma_{\text {bulk }}$ value.

The large SP areas can also not be explained by an ordered surface film with a structure different from that of

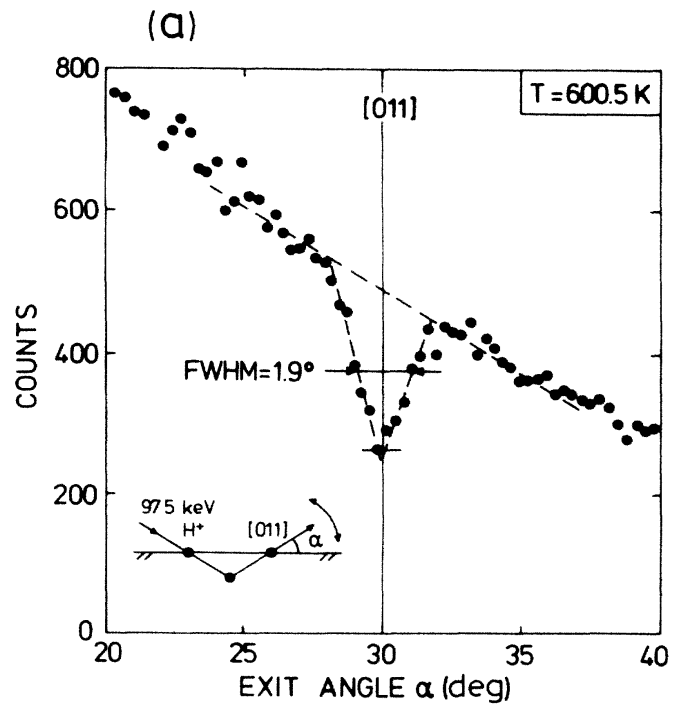

(b)

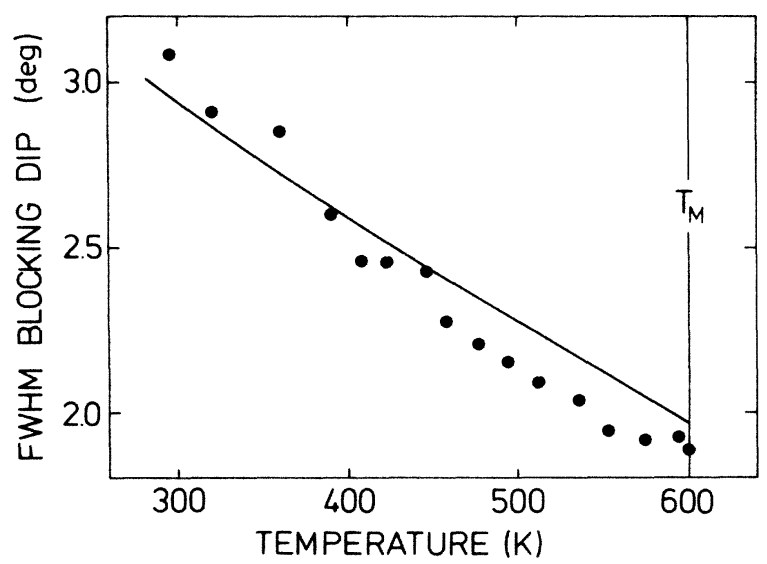

FIG. 6. (a) [011] blocking dip from the bulk of $\mathrm{Pb}$, measured at backscattering energies ranging from 92 to $93 \mathrm{keV}$. The inset shows the scattering geometry (see also Fig. 2). (b) Measured width (FWHM) of the [011] bulk blocking dip as a function of temperature. The solid curve is discussed in the text. the underlying substrate (e.g., rotated). This would lead to blocking or flux-peaking effects in the angular distribution of the SP area, even for a rotated surface film. As mentioned before, such effects are not observed close to $T_{m}$ (Fig. 5). The only way to make the simulation fit the area and shape of the energy spectra close to $T_{m}$, is to keep $\sigma_{\text {bulk }}$ at $0.28 \AA$ and to have a thick slab, e.g., $\sim 16$ monolayers at $600.5 \mathrm{~K}$, of disorderly positioned (fully visible) $\mathrm{Pb}$ atoms covering an ordered $\mathrm{Pb}$ substrate.

To illustrate the sensitivity of our measurements to disorder, Monte Carlo simulations have been performed for crystals in which only the atoms in a limited depth region were given an enhanced rms displacement $\sigma_{s}$, while the deeper layers were kept at $\sigma_{\text {bulk }}=0.28 \AA$. The position distribution of atoms around the lattice points was assumed to be Gaussian. In order to fit the energy spectrum measured at $581 \mathrm{~K}$ the atoms in the outermost $\sim 8 \mathrm{~Pb}$ monolayers must have an rms displacement of $\sigma_{s} \geq 0.6 \AA$. For a fit to the spectrum at $600.5 \mathrm{~K}$ the atoms in the top $\sim 16$ monolayers must have an $\mathrm{rms}$ displacement of $\sigma_{s} \geq 1.0 \AA$. These $\sigma_{s}$ values are very high compared to the bulk vibration amplitude of $0.28 \mathrm{~A}$, and lead to a considerable overlap between the position distributions of nearest-neighbor atoms. In case of a $\sigma_{s}$ value of $1.0 \AA$, the probability density for atoms to be halfway between two nearest-neighbor lattice points (the nearest-neighbor distance in $\mathrm{Pb}$ is $3.5 \AA$ ) would already be $43 \%$ of the maximum probability density at a lattice point.

At somewhat lower temperatures, e.g., $597 \mathrm{~K}$, the SP does not yet have the simple shape of Fig. 2(b), but the SP area already has an anomalously high intensity. The changes in the shape of the energy spectra with temperature were analyzed to determine the temperature $T^{*}$ at which the formation of a completely disordered film starts. If the difference between the energy spectrum at $T^{*}$ and each of the higher-temperature spectra is exclusively caused by an additional number of molten layers at high temperatures, it should be possible to construct all higher-temperature spectra by addition of a "molten film spectrum" $(M)$ to a copy of the substrate spectrum $(S)$ at $T^{*}$, accordingly shifted in energy, as suggested in Fig. 2(b). Of course, each energy spectrum above $T^{*}$ would do equally well as substrate spectrum, all differences between spectra above $T^{*}$ being the result of differences in melt depth. $T^{*}$ is the lowest temperature for which this adding procedure should result in good fits to highertemperature spectra. A molten film spectrum consists of a rectangular block having the width of the molten film [Fig. 2(b)] and a height of 1, convoluted with the Gaussian detector function, and, at lower energies, a multiple scattering contribution due to random deflections of protons in the molten film. The outlined procedure is illustrated in Figs. $7(\mathrm{a})$ and $7(\mathrm{~b})$, where the energy spectra measured at 592 and $515 \mathrm{~K}$ are subsequently used as the substrate spectrum with which a fit to the $600.5-\mathrm{K}$ spectrum is attempted. In both cases the molten film thickness has been optimized to give the best result. Clearly, this yields a good fit only for $592 \mathrm{~K}$, i.e., spectrum differences can be fully ascribed to differences in melt depth between 600.5 and $592 \mathrm{~K}$, but not between 600.5 and $515 \mathrm{~K}$. In this way the differences between the spectrum at 600.5 


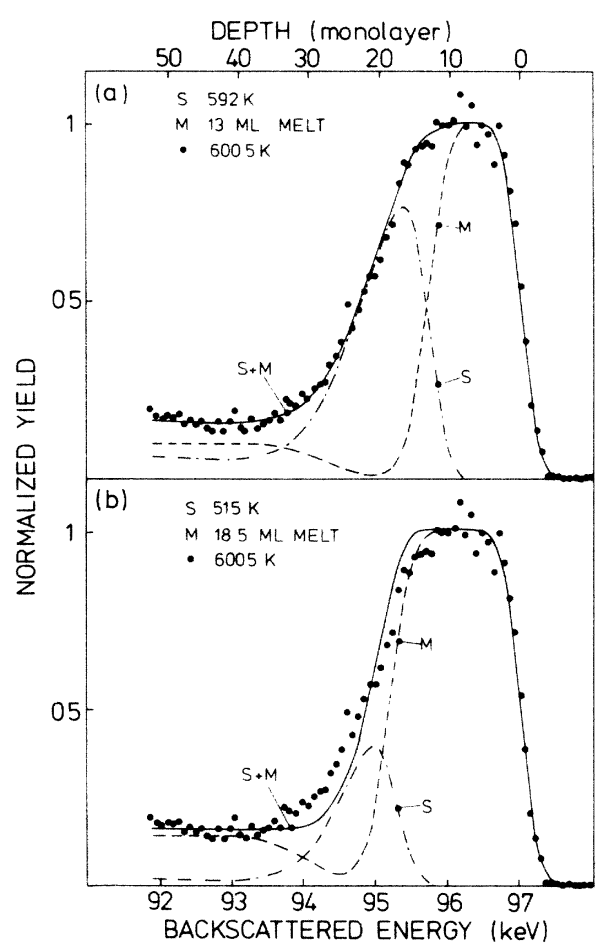

FIG. 7. Fits to the spectrum at $600.5 \mathrm{~K}$, obtained by adding a calculated molten film spectrum $M$ to a shifted copy of the substrate spectrum $S$ measured at (a) $592 \mathrm{~K}$, and (b) $515 \mathrm{~K}$.

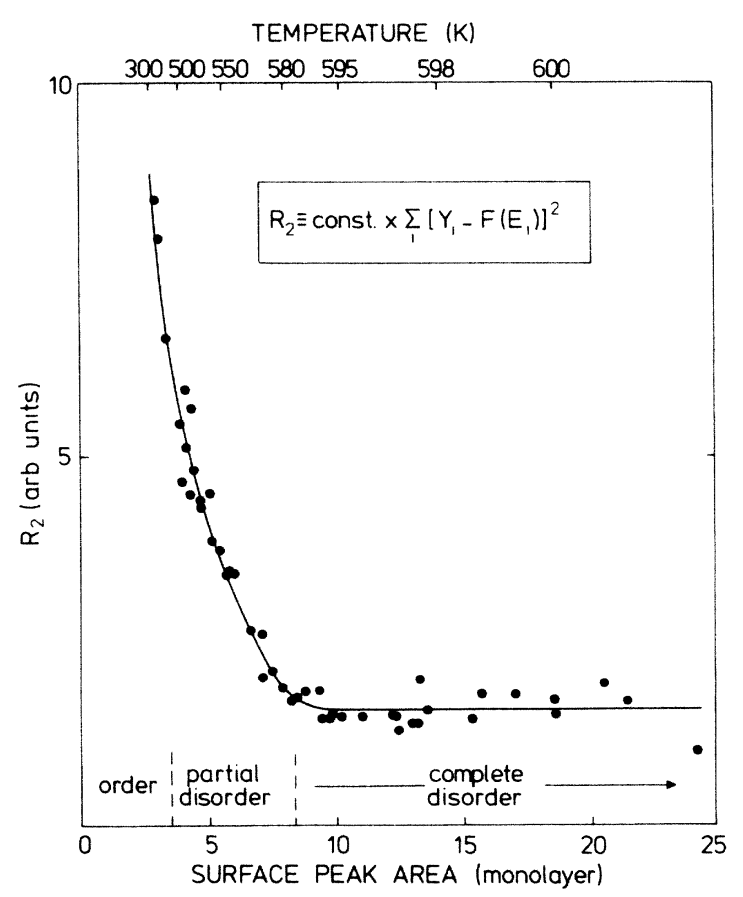

FIG. 8. Goodness-of-fit parameter $R_{2}$ of the fitting procedure discussed in the text and illustrated in Fig. 7, versus the surface-peak area.
$\mathbf{K}$ and all lower-temperature spectra have been examined. The resulting goodness-of-fit parameter $R_{2}$, defined as the average squared difference between a fit and the $600.5-\mathrm{K}$ spectrum, is displayed in Fig. 8. Down to $T^{*}=580 \mathrm{~K}$ all $R_{2}$ values are equally low, so all spectrum differences above $580 \mathrm{~K}$ can be attributed to a growth of molten film thickness. Melt depths obtained in this way are indicated on the right-hand vertical axis of Fig. 4.

The $T^{*}$ value of $580 \mathrm{~K}$ is an upper limit for the temperature at which the surface becomes completely disordered. The analysis in Figs. 7 and 8 is possibly too restrictive, in that it assumes the molten film thickness to be the only parameter changing with temperature. If the substrate (spectrum $S$ ) were to change too, simultaneously with the melt depth, the above procedure would have yielded unacceptable $R_{2}$ values. $T^{*}$ is the temperature from whereon only the melt depth changes, and the substrate remains the same.

Figure 4 shows that at $580 \mathrm{~K}$ the SP area already exceeds curve II by $\sim 5$ monolayers of visible $\mathrm{Pb}$ atoms. The large $R_{2}$ values below $580 \mathrm{~K}$ (Fig. 8) indicate that these extra visible atoms cannot be described simply in terms of a fully disordered overlayer. In Fig. 9 the SP width is plotted versus the SP area, in units of monolayers. The conversion into monolayers involved a deconvolution of the resolution function of the energy analyzer $(\delta E=390 \mathrm{eV})$ from the SP width, and then a division by the random stopping power of $96 \mathrm{eV} /$ monolayer (equivalent to $13.7 \mathrm{eV} / \mathrm{A}$ path length ${ }^{35}$ ). The solid line in Fig. 9 indicates the expected linear relation between SP width and SP area, ${ }^{47}$ starting with zero width at 1 visible monolayer and having a derivative of 1 . At low temperatures the SP width increases much more rapidly (and the SP height is correspondingly lower) than expected. The large SP widths can be obtained in a simulated energy

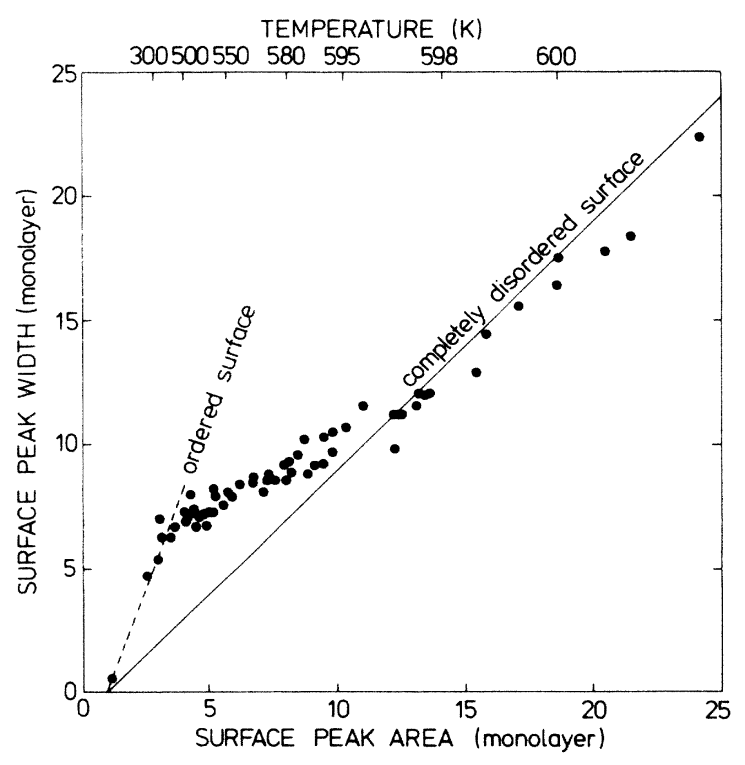

FIG. 9. Surface-peak width versus surface-peak area. Indicated is the behavior for a well-ordered (dashed line) and a completely disordered surface (solid line). The lowest point has been measured at $29 \mathrm{~K}$ (Ref. 43). 
spectrum only by assuming the stopping power to be increased with respect to the random value by a factor of $2.50 \pm 0.25$. This enhancement is related to the alignment of proton beam and detector with major crystal axes. In order for protons to scatter from atoms in, e.g., the fifth layer, and to be subsequently detected, they must travel along a $\langle 110\rangle$ row and pass four atoms at relatively short distance, both on their way in and on their way out. The average electron density traversed by these protons, and thus the average energy loss they suffer, ${ }^{48,49}$ is accordingly higher than it would be for a randomly oriented proton beam and detector. It was checked that upon misorientation of the crystal the observed stopping power indeed returned to its random value.

As an enhancement of the stopping power arises only in case of well-defined atom rows (or planes), it can be regarded as a "fingerprint" of crystallinity. The stopping power is therefore highly sensitive to disorder in the surface region contributing to the SP. This is illustrated in Fig. 9 where the plotted SP width exhibits a kink at 3.5 visible $\mathrm{Pb}$ layers. Note, that this corresponds closely to the temperature of $450 \mathrm{~K}$ where the data in Fig. 4 start deviating from the behavior calculated for a well-ordered surface (curve II). Between $450 \mathrm{~K}$ and $\sim 597 \mathrm{~K}$ the SP width increases at a strongly reduced rate, implying that the additional atoms becoming visible in this temperature range are displaced far out from the $\langle 110\rangle$ rows. At these temperatures the surface region is only partially disordered.

From the results presented in Figs. 4, 8, and 9, the following model of the melting transition emerges. Up to $450 \mathrm{~K}$ the surface remains well ordered. Then the nearsurface layers become partially disordered. From $580 \mathrm{~K}$ onward, a completely disordered film builds up with increasing thickness, on top of the partially disordered surface region. The latter forms the transition between the well-ordered substrate and the fully disordered film, and contains about five extra visible layers over a depth range in the order of $\sim 10$ monolayers.

The above picture represents both a time average (measuring time of $\sim 30 \mathrm{sec}$ per spectrum), and a spatial average (beam spot size of $0.5 \times 1.0 \mathrm{~mm}^{2}$ ). It is therefore not possible to decide from the SP shapes whether the instantaneous structure of the transition layer would be some mixture of solid and molten grains, or a laterally smooth change from solid to melt (see Secs. VIA, VIC, and VID).

\section{RHEED}

One of the earlier experimental searches for surface melting, which is often referred to, is a LEED study by Goodman and Somorjai. ${ }^{50}$ They examined the temperature dependence of diffraction spot intensities from the low-index surfaces of $\mathrm{Pb}, \mathrm{Bi}$, and $\mathrm{Sn}$. For each surface studied, the diffraction pattern was found to remain visible up to the very (bulk) melting point, the spot intensities decreasing with temperature in accordance with the expected Debye-Waller factor. This was interpreted as proof against a lowered surface melting point. The contradiction between this LEED work and the ion scattering results reported in the previous section, prompted us to in- vestigate the $\mathrm{Pb}(110)$ surface near $T_{m}$ with electron diffraction too. Straylight from the filament with which the sample was heated, made LEED observations difficult at high temperatures. This problem was circumvented by using the glancing diffraction angle configuration of a RHEED setup.

Figure 10 shows a series of RHEED patterns, obtained with $12-\mathrm{keV}$ electrons, incident along the [001] azimuth, at an angle of $\sim 2^{\circ}$ with the $\mathrm{Pb}(110)$ surface plane. The temperature ranges from room temperature up to just below $T_{m}$. At low temperatures [Figs. 10(a) and 10(b)] the patterns contain three diffraction contributions: (i) strong diffraction rods reflecting surface periodicity in the [11̄0] direction; (ii) Kikuchi bands and lines, produced by inelastically scattered electrons diffracting from bulk lattice planes; ${ }^{51}$ and (iii) weak bulk diffraction spots due to macroscopic $\sim 1^{\circ}$ undulations of the surface, which are related to the preparation of the sample.

As the temperature is raised from 295 to $475 \mathrm{~K}$ the diffraction intensities decrease somewhat, while the background comes up [Figs. 10(a)-10(c)]. Above $475 \mathrm{~K}$ the diffraction intensities fall off at an increased rate (Figs. 10(d)-10(f)]. At $565 \mathrm{~K}$ the RHEED pattern shows absolutely no sign of any surface diffraction rod [Fig. 10(g)]. Note, that at the same temperature the pattern still shows weak Kikuchi bands, reflecting the persistence of welldefined bulk lattice planes. Finally, at higher temperatures [Fig. 10(h)] also the Kikuchi bands disappear. Similarly to the ion scattering data, the RHEED patterns are constant in time and reversible with temperature.

From these RHEED observations we conclude that at $565 \mathrm{~K}$ the depth range probed by the surface-diffracted electrons ( $\sim 3$ monolayers) is disordered, while the bulk retains its normal crystal structure. The temperature dependence of the intensity of the diffraction rods suggests that surface disordering is a gradual process, extending over a temperature range of at least $100 \mathrm{~K}$. The striking difference between these results and those reported by Goodman and Somorjai ${ }^{50}$ remains puzzling.

\section{INFRARED EMISSIVITY}

Finally, we present indirect evidence for a change in electronic behavior at the $\mathrm{Pb}(110)$ surface close to $T_{m}$. For several materials the optical constants are known to jump at the melting point. ${ }^{52-57}$ This phenomenon is related to the sudden decrease of the relaxation time of the conduction electrons at $T_{m}$, as a result of the loss of lattice periodicity. This affects the complex dielectric constant, and thereby properties such as the electrical and thermal conductivities [both decreasing by $\sim 50 \%$ for $\mathrm{Pb}$ (Ref. 31)] and the optical constants. ${ }^{52,56}$

Figure 11 shows the temperature dependence of the infrared emissivity $\epsilon$ of $\mathrm{Pb}(110)$, for wavelengths between 2.0 and $2.6 \mu \mathrm{m}$, measured with an Ircon-300C pyrometer. $\mathrm{Up}$ to $\sim 595 \mathrm{~K} \epsilon$ changes only little with temperature. Above $595 \mathrm{~K} \epsilon$ rises, at first slowly, and within $0.1 \mathrm{~K}$ from $T_{m}$ very rapidly, up to $\sim 1.5$ times the original $\epsilon$ value. ${ }^{58}$ This behavior is reversible with temperature.

Figure 11 suggests that close to $T_{m}$ the emitted infrared radiation has a high- $\epsilon$ contribution from the molten surface film, and a low- $\epsilon$ substrate contribution, which is 

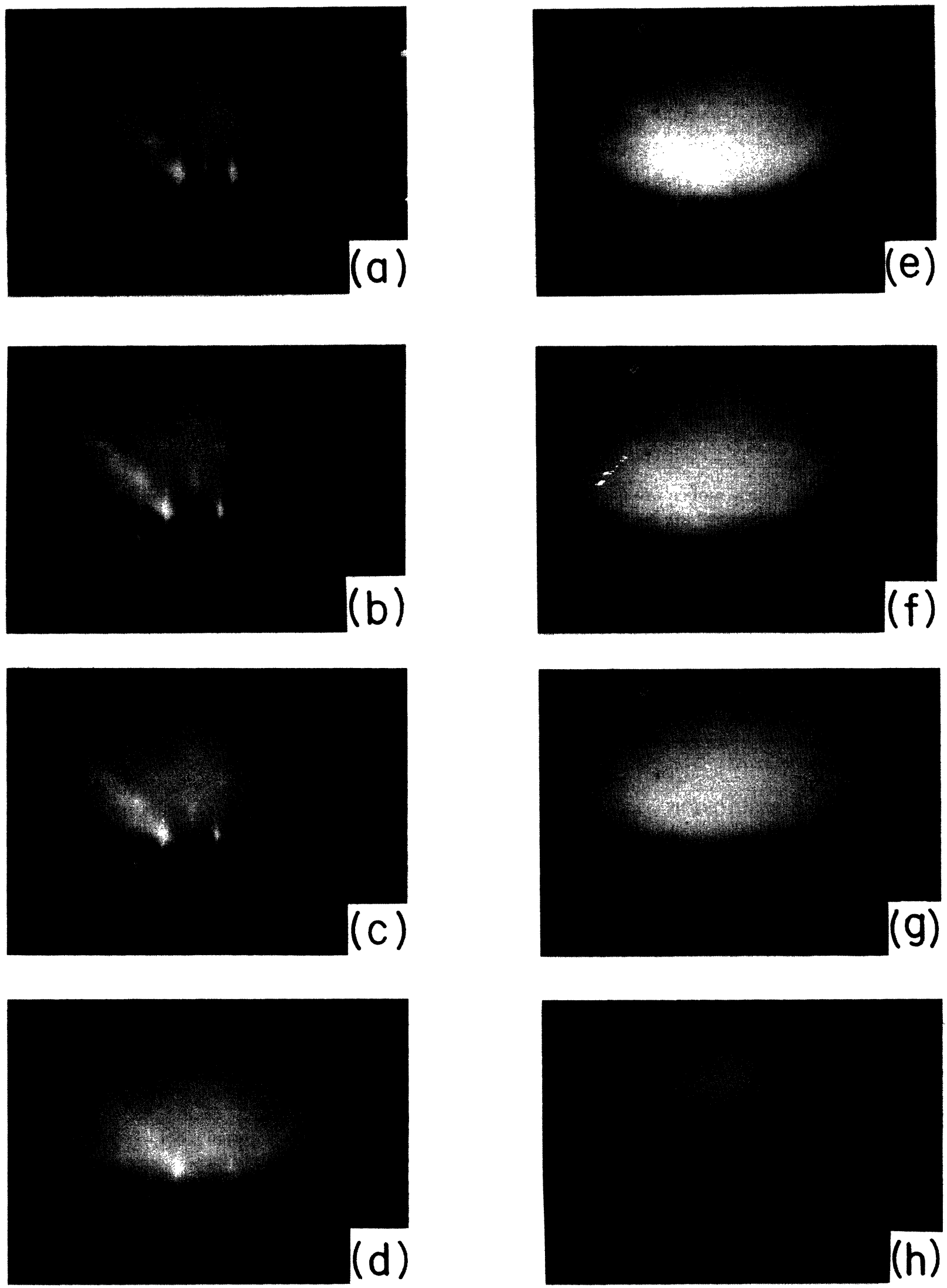

FIG. 10. RHEED patterns obtained at different temperatures: (a) $295 \mathrm{~K}$; (b) $370 \mathrm{~K}$; (c) $475 \mathrm{~K}$; (d) $532 \mathrm{~K}$; (e) $551 \mathrm{~K}$; (f) $558 \mathrm{~K}$; (g) $565 \mathrm{~K}$; and (h) just below $T_{m}$. 


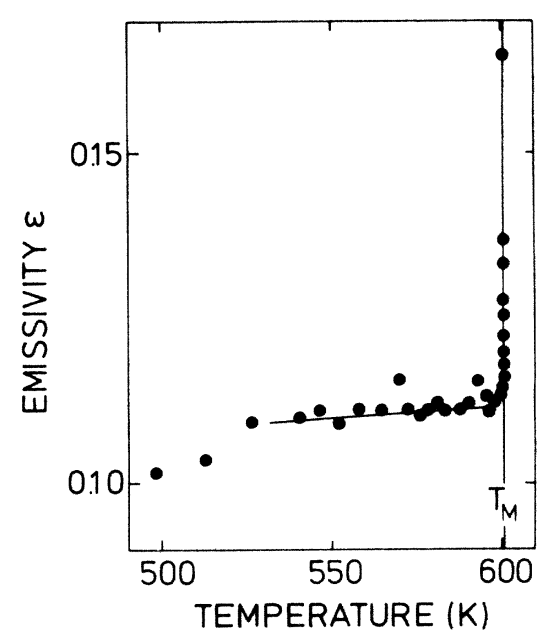

FIG. 11. Measured infrared emissivity (for 2.0-2.6 $\mu \mathrm{m}$ wavelength) versus temperature.

weakened by absorption in the molten layer. The ratio between the two contributions depends on the layer thickness (and on the absorption coefficient of the melt).

Similar observations of a rise in $\epsilon$ below $T_{m}$ have been reported earlier for thin $\mathrm{Ga}$ films ${ }^{56,57}$ and for spherical $\mathrm{Cu}$ crystals. ${ }^{53-55}$ The observations on $\mathrm{Cu}$ were interpreted as an indication of surface melting. Precise knowledge of the optical constants of liquid and solid $\mathrm{Cu},{ }^{52}$ close to $T_{m}$ allowed for a rough estimate of the liquid film thickness in that case. Throughout this paper, measurements of the surface temperature with the infrared pyrometer have been corrected for the temperature dependence of $\epsilon$ in Fig. 11.

\section{DISCUSSION}

\section{A. Surface roughening}

At this point we stress that the surface melting transition reported in this paper is different from the surface roughening transition, proposed by Burton, Cabrera and Frank. 59 These authors called the latter transition "surface melting," which has caused considerable confusion in the literature since then. In case of surface roughness all atoms still occupy well-defined crystal lattice positions but the crystal-vacuum (vapor) interface is rough on an atomic scale. ${ }^{3}$ Although surface roughening is not expected to take place at low-index metal crystal surfaces, ${ }^{3,60}$ recent helium scattering experiments are suggestive of an effect on $\mathrm{Ni}(110)$ (Ref. 61) and $\mathrm{Cu}(110){ }^{62}$ The ion scattering data of Sec. III are insensitive to surface roughness. In a rough surface the atoms, still residing at lattice positions, would shadow each other just as well as in a flat surface, and blocking effects would even be stronger. The disorder observed in the present experiment is of a different type, since it involves atoms detached from lattice positions. Roughening is expected to play a role at the solid-liquid interface; this follows from a theory due to Jackson, ${ }^{60}$ who relates the occurrence of interface roughness to the latent heat of melting and the packing density of the crystal face in contact with the melt. The transition layer, which builds up in the temperature region between 450 and $580 \mathrm{~K}$, before a continuous molten film forms, might well be the depth region covering such interface roughness.

\section{B. Related experiments}

Closely related to the experiments described here is a recent $x$-ray diffraction and differential scanning calorimetry study of the melting behavior of thin $\mathrm{Pb}$ films sandwiched between Ge layers. ${ }^{63}$ For these $\mathrm{Pb}$ films the melting transition was found to start tens of degrees below $T_{m}$, and to exhibit an appreciable temperature breadth. These findings can be understood in terms of surface melting, insofar as the $\mathrm{Pb}-\mathrm{Ge}$ interface and/or the grain boundaries within the $\mathrm{Pb}$ films can be regarded as similar to the $\mathrm{Pb}$-vacuum interface. Not only thin films, ${ }^{56,57,63}$ but also small particles ${ }^{64,65}$ tend to have melting points far below $T_{m}$.

Many papers have been devoted to the formation of quasiliquid layers observed on ice crystals. ${ }^{66-71}$ Of particular interest is a proton channeling investigation by Golecki and Jaccard. ${ }^{69}$ These authors found a strongly disordered surface region of appreciable thickness $(\sim 1000$ $\AA, 1 \mathrm{~K}$ below $T_{m}$ ) on the basal plane of ice. However, in this quasiliquid surface layer partial order is retained. The polar water molecules line up in a preferred orientation at the water surface thereby making such a film energetically quite favorable. ${ }^{70}$ It is this additional driving force for surface melting on ice (and other hydrogenbonded solids ${ }^{70}$ ) that makes the resulting quasiliquid films so thick. At a metal surface such orientation effects do not exist. Therefore, the surface melting effect on $\mathrm{Pb}(110)$ is of different nature.

\section{Computer experiments}

In most molecular dynamics (MD) studies of the melting behavior of crystal slabs or semi-infinite crystals ${ }^{15-20}$ Lennard-Jones pair potentials have been employed to model the interactions between atoms. Despite the shortcomings of such a description in case of metal crystals and their surfaces, the results of these computer experiments strongly resemble the experimental observations for $\mathrm{Pb}(110) .^{72}$ These computer simulations exhibit a surface melting effect, which, as in our case, starts with a gradual disordering of the surface region and finally results in a liquidlike surface film with some transition layer between this film and the ordered substrate. ${ }^{15}$ The MD studies show only a weak surface crystallographic dependence, i.e., nearly equal liquid film thicknesses are found on different crystal faces.

\section{Theory}

Two recent theoretical approaches to (surface) melting are mentioned. In the first, the temperature is calculated at which a vibrational lattice instability occurs at a crystal surface. ${ }^{12-14}$ This approach actually transfers the well known "Lindemann criterion" to the solid surface. ${ }^{21}$ Lin- 
demann ${ }^{4}$ showed that melting occurs when the vibration amplitude reaches a certain critical fraction $(\sim 10 \%)$ of the interatomic distance. The surface instability temperature is found to be lower than the corresponding temperature in the bulk. ${ }^{12-14}$ It is also lower than the melting temperature, at which the instability begins to propagate into the bulk. This theory thus predicts the possibility of having a stable liquidlike layer on the surface of a solid below the bulk melting point. In addition it shows that the interior of the solid may be superheated when the influence of the surface melt is avoided. ${ }^{23-27}$ Inclusion of multilayer relaxation effects in the theory leads to more stable surfaces, and reverses the order in which the lowindex surfaces of metals are predicted to become unstable, from (110)-(100)-(111) to (111)-(100)-(110). ${ }^{13}$ Neglecting relaxation effects, the surface instability is found at $0.75 T_{m}$, which corresponds well with the measured onset of partial surface disordering for $\mathrm{Pb}(110)$.

Finally, we turn to the theoretical treatment of semiinfinite systems with first-order bulk transitions by Lipowsky and Speth. ${ }^{10,11}$ Minimizing the Landau expression for the free energy of a semi-infinite system, these authors calculate order parameter profiles as a function of distance from the surface for temperatures close to the bulk transition temperature $T_{m}$. Depending on the choice of the Landau coefficients in the energy expression, several types of phase transitions are possible. In some of these the surface order parameter decreases continuously to zero as the temperature approaches $T_{m}$. A disordered surface layer is formed, separated from the ordered bulk by a delocalized (i.e., diffuse) interface. The thickness $l$ of this surface layer (including half of the interface) is given by

$$
l=l_{0} \ln \left[T_{0} /\left(T_{m}-T\right)\right],
$$

where $T_{m}$ is approached from below. The constant $T_{0}$ specifies the temperature difference $T_{m}-T$ at which the buildup of a disordered film starts. The scaling length $l_{0}$ is the correlation length within the disordered phase. The inset in Fig. 4 shows that our measurements are indeed consistent with such behavior, with $l_{0}=6.23 \AA(=3.56$ monolayer) and $T_{0}=55 \mathrm{~K}^{73}$ Note, that $l_{0}$ is of microscopic dimension (between one and two times the nearestneighbor distance), as expected for a system governed by short-range forces. ${ }^{11}$

\section{CONCLUSION}

Melting of a $\mathrm{Pb}$ crystal begins at the surface. The data presented in this paper show for $\mathrm{Pb}(110)$ that surface melting is a reversible and continuous process. The surface starts to disorder partially at a temperature which is $\frac{3}{4}$ of the bulk melting temperature $T_{m}$. At $20 \mathrm{~K}$ below $T_{m}$, the topmost surface layer becomes fully disordered, as seen by ion shadowing and blocking measurements. The thickness of the disordered layer diverges logarithmically as the melting point is approached. These observations are consistent with recent theoretical predictions.

\section{ACKNOWLEDGMENTS}

The authors express their gratitude to A. J. Riemersma and P. H. M. van Berge Henegouwen of the University of Amsterdam for their invaluable contributions to the preparation of our $\mathrm{Pb}$ specimens. Dr. F. W. Saris is gratefully acknowledged for stimulating discussions and a critical reading of the manuscript. This work is part of the research program of the Stichting voor Fundamenteel Onderzoek der Materie (Foundation for Fundamental Research on Matter) and was made possible by financial support from the Nederlandse Organisatie voor ZuiverWetenschappelijk Onderzoek (Netherlands Organization for the Advancement of Pure Research).
${ }^{1}$ A. R. Ubbelohde, The Molten State of Matter (Wiley, New York, 1978), and references therein.

${ }^{2}$ R. M. Cotterill, E. J. Jensen, and W. D. Kristensen, in Anharmonic Lattices, Structural Transitions and Melting, edited by T. Riste (Noordhoff, Leiden, 1974), and references therein.

${ }^{3} \mathrm{D}$. P: Woodruff, The Solid-Liquid Interface (Cambridge University Press, Cambridge, 1973), and references therein.

${ }^{4}$ F. A. Lindemann, Z. Phys. 14, 609 (1910).

${ }^{5}$ M. Born, J. Chem. Phys. 7, 591 (1939).

${ }^{6} \mathrm{~J}$. Frenkel, Kinetic Theory of Liquids (Dover, New York, 1955).

${ }^{7}$ J. E. Lennard-Jones and A. F. Devonshire, Proc. R. Soc. London, Ser. A 170, 464 (1939).

${ }^{8}$ D. Kuhlmann-Wilsdorf, Phys. Rev. A 140, 1599 (1965).

${ }^{9}$ J. K. Kristensen and R. M. J. Cotterill, Philos. Mag. 36, 437 (1977).

${ }^{10}$ R. Lipowsky, Phys. Rev. Lett. 49, 1575 (1982); R. Lipowsky and W. Speth, Phys. Rev. B 28, 3983 (1983).

${ }^{11}$ R. Lipowsky (unpublished).

${ }^{12}$ L. Pietronero and E. Tosatti, Solid State Commun. 32, 255 (1979).
${ }^{13}$ C. S. Jayanthi, E. Tosatti, and A. Fasolino, Surf. Sci. 152/153, 155 (1985).

${ }^{14}$ C. S. Jayanthi, E. Tosatti, and L. Pietronero, Phys. Rev. B 31, 3456 (1985).

${ }^{15}$ J. Q. Broughton and G. H. Gilmer, J. Chem. Phys. 79, 5105 (1983); 79, 5119 (1983).

${ }^{16}$ F. F. Abraham, Phys. Rev. B 23, 6145 (1981).

17J. Q. Broughton and G. H. Gilmer, Acta Metall. 31, 845 (1983).

${ }^{18} \mathrm{~W}$. Schommers and P. von Blanckenhagen, Vacuum 33, 733 (1983).

${ }^{19} \mathrm{~W}$. Schommers and P. von Blanckenhagen, Surf. Sci. 162, 144 (1985).

${ }^{20}$ R. M. J. Cotterill, Philos. Mag. 32, 1283 (1975).

${ }^{21}$ P. R. Couchman and W. A. Jesser, Philos. Mag. 35, 787 (1977).

${ }^{22}$ G. Tammann, Z. Phys. 11, 609 (1910).

${ }^{23}$ V. I. Motorin and S. L. Musher, J. Chem. Phys. 81, 465 (1984).

${ }^{24}$ M. Kass and S. Magun, Z. Kristallogr. 116, 354 (1961). 
${ }^{25}$ S. E. Haikin and V. P. Bene, Dokl. Akad. Nauk SSSR 23, 31 (1939).

${ }^{26}$ R. L. Cormia, J. D. Mackenzie, and D. Turnbull, J. Appl. Phys. 34, 2239 (1963).

${ }^{27}$ N. G. Ainslie, J. D. Mackenzie, and D. Turnbull, J. Phys. Chem. 65, 1718 (1961).

${ }^{28} \mathrm{G}$. Lewin, Fundamentals of Vacuum Science and Technology (McGraw-Hill, New York, 1965).

${ }^{29}$ W. J. McG. Tegart The Electrolytic and Chemical Polishing of Metals in Research and Industry (Pergamon, New York, 1959).

${ }^{30} \mathrm{M}$. Hansen and K. Anderko, Constitution of Binary Alloys (McGraw-Hill, New York, 1985); R. E. Elliot, Constitution of Binary Alloys, First Supplement (McGraw-Hill, New York, 1985); F. A. Shunk, Constitution of Binary Alloys, Second Supplement (McGraw-Hill, New York, 1985).

${ }^{31}$ Zahlenwerte und Funktionen aus Physik, Chemie, Astronomie, Geophysik und Technik, Vol. 4 of Landolt-Börnstein, edited by $\mathrm{H}$. Borchers and E. Schmidt (Springer-Verlag, Berlin, 1964).

32A. R. Miedema, Z. Metallkd. 69, 455 (1978).

${ }^{33}$ A. R. Miedema, Z. Metallkd. 69, 287 (1978).

34J. F. van der Veen, Surf. Sci. Rep. 5, 199 (1985).

${ }^{35}$ H. H. Andersen and J. F. Ziegler, The Stopping and Ranges of Ions in Matter (Pergamon, New York, 1977), Vol. 3.

36J. W. M. Frenken, J. F. van der Veen, R. N. Barnett, U. Landman, and C. L. Cleveland, Surf. Sci. 172, 319 (1986)

${ }^{37}$ The result of a convolution of a Gaussian with a block function reaches a maximum height of $98 \%$ of the block height, when the block width is 2 times the FWHM of the Gaussian.

${ }^{38}$ Spectrum (g) in Fig. 3 shows a weak slope as a result of the $5 \%$ variation in width of the resolution function of the energy analyzer over the plotted energy range.

${ }^{39}$ J. W. M. Frenken, R. M. Tromp, and J. F. van der Veen, Nucl. Instrum. Methods (to be published).

${ }^{40}$ J. H. Barrett, Phys. Rev. B 3, 1527 (1971).

${ }^{41}$ E. V. Zarochentsev, S. P. Kravchuk, and T. M. Tarusina, Fiz. Tverd. Tela (Leningrad) 18, 43 (1976) [Sov. Phys.-Solid State 18, 239 (1976)], and refs. therein.

${ }^{42} \mathrm{~A}$ surface blocking analysis of $\mathrm{Pb}(110)$ at $450 \mathrm{~K}$ also supports the conclusion that the surface is well-ordered at this temperature (see Ref. 43).

${ }^{43}$ J. W. M. Frenken, F. Huussen, and J. F. van der Veen (unpublished).

${ }^{44}$ D. V. Morgan, Channeling (Wiley, London, 1973).

${ }^{45}$ In a previously published version of Fig. 4 the right-hand vertical axis was shifted by 1.6 monolayers (see Ref. 46).

46J. W. M. Frenken and J. F. van der Veen, Phys. Rev. Lett. 54, 134 (1985).

${ }^{47}$ The linear relation in Fig. 9 assumes a Gaussian intrinsic shape of the SP. Other SP shapes lead to a slightly modified curve.

${ }^{48}$ I. Nagy, J. Lászlo, and J. Giber, Nucl. Instrum. Methods B
$15,8(1986)$

${ }^{49}$ K. B. Winterbon, Radiat. Eff. 79, 251 (1983).

${ }^{50}$ R. M. Goodman and G. A. Somorjai, J. Chem. Phys. 52, 6325 (1970).

${ }^{51}$ P. B. Hirsch, Electron Microscopy of Thin Crystals (Krieger, New York, 1977).

${ }^{52}$ M. Otter, Z. Phys. 161, 539 (1961).

${ }^{53}$ K. D. Stock, Surf. Sci. 91, 655 (1980).

${ }^{54}$ K. D. Stock and E. Menzel, J. Cryst. Growth 43, 135 (1978).

${ }^{55}$ K. D. Stock and E. Menzel, Surf. Sci. 61, 272 (1976).

${ }^{56} \mathrm{P}$. Cheyssac, R. Kofman, and R. Carrigos, Solid State Commun. 44, 1583 (1982).

${ }^{57}$ R. Kofman, P. Cheyssac, and R. Carrigos, J. Phys. F 9, 2345 (1979).

${ }^{58}$ Temperatures measured with the pyrometer were corrected for the increase in emissivity close to melting (Sec. II). In a previous report of the surface-melting observation (Ref. 46) a constant emissivity was assumed, which introduced minor $(<2$ $\mathrm{K}$ ) errors in the temperature scale for temperatures above $\mathbf{5 8 0}$ K.

${ }^{59}$ W. Burton, N. Cabrera, and F. C. Frank, Philos. Trans. R. Soc. London, Ser. A 243, 299 (1951).

${ }^{60} \mathrm{~K}$. A. Jackson, in Progress in Solid State Chemistry, edited by H. Reiss (Pergamon, New York, 1967), Vol. 4.

${ }^{61}$ M. den Nijs, E. K. Riedel, E. H. Conrad and T. Engel, Phys. Rev. Lett. 55, 1689 (1985).

${ }^{62}$ D. Gorse and J. Lapujoulade, Surf. Sci. 162, 847 (1985).

${ }^{63}$ R. H. Willens, A. Kornblit, L. R. Testardi, and S. Nakahara, Phys. Rev. B 25, 290 (1982).

${ }^{64}$ Gl. S. Zhdanov, Kristallografiya 21, 1220 (1976) [Sov. Phys. Crystallogr. 21, 706 (1976)].

${ }^{65}$ S. J. Peppiatt and J. R. Sambles, Proc. R. Soc. London, Ser. A 345,387 (1975).

${ }^{66}$ N. H. Fletcher, Surf. Sci. 115, L103 (1982).

${ }^{67}$ D. Nason, Surf. Sci. 102, L7 (1981).

${ }^{68}$ D. Beaglehole and D. Nason, Surf. Sci. 96, 357 (1980).

${ }^{69}$ I. Golecki and C. Jaccard, J. Phys. C 11, 4229 (1978).

${ }^{70}$ D. Nason, J. Chem. Phys. 64, 3930 (1976).

${ }^{71}$ D. Nason and N. H. Fletcher, J. Chem. Phys. 62, 4444 (1975).

${ }^{72}$ Preliminary MD simulations of surface melting of simple metals with use of pairpotentials obtained from pseudopotential theory, volume energy, and single-ionic contributions indicate the occurrence of surface premelting in the form of 1 to 3 disordered layers, which, at temperatures close to melting, extend to approximately 10 liquefying layers: R. N. Barnett, U. Landman, and C. L. Cleveland (private communication).

${ }^{73}$ The melt depths on the right-hand vertical axis of Fig. 4 do not include half the thickness of the transition layer (see Sec. III C). For the calculation of $l_{0}$ from the experimental data an estimated half-width of 5 monolayers was therefore added to the melt depths from Fig. 4. This only affects the value of $T_{0}$ in Eq. (6.1) $\left(T_{0}=13.5 \mathrm{~K}\right.$ neglecting the half-width), and leaves $l_{0}$ unchanged. 

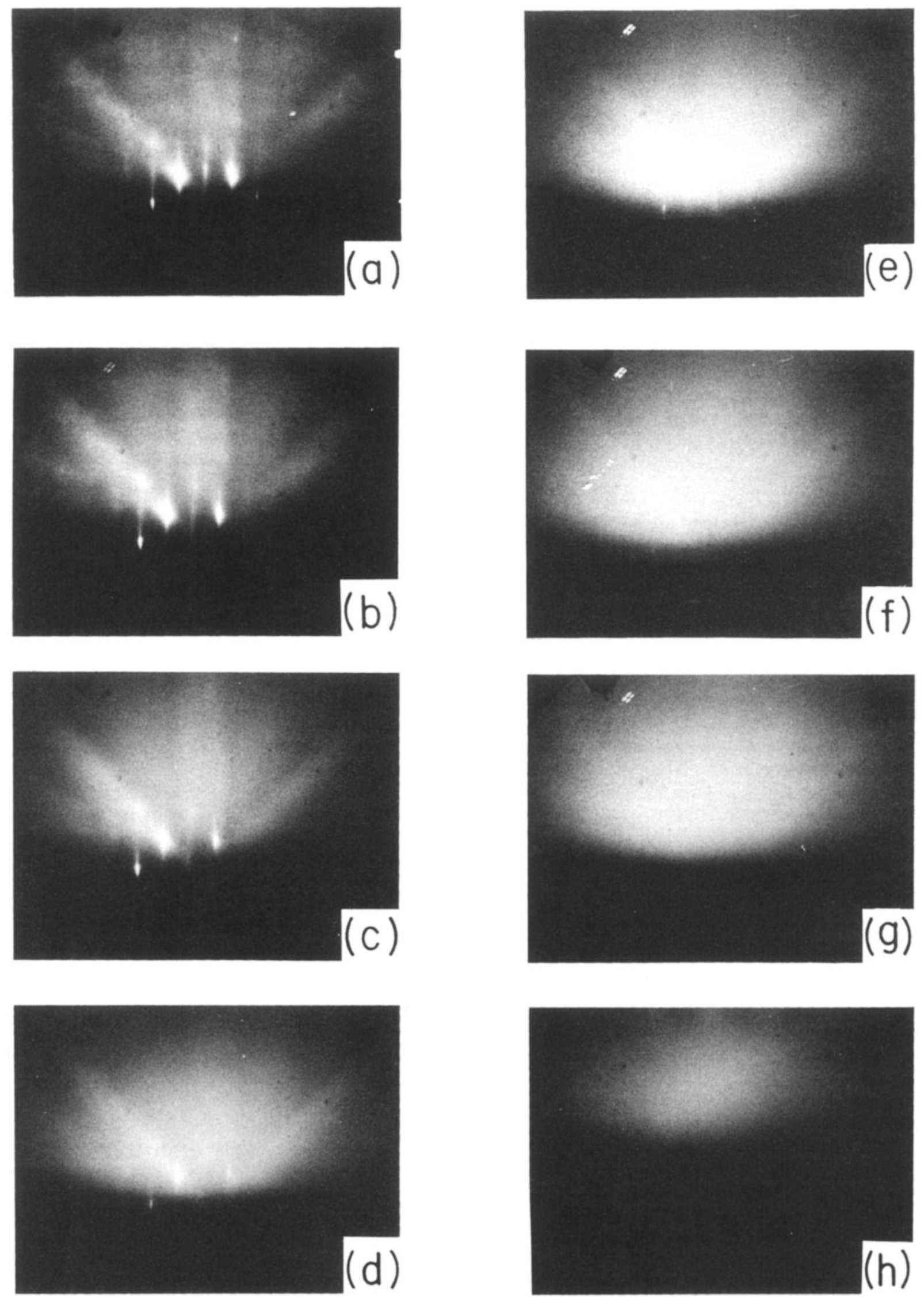

FIG. 10. RHEED patterns obtained at different temperatures: (a) $295 \mathrm{~K}$; (b) $370 \mathrm{~K}$; (c) $475 \mathrm{~K}$; (d) $532 \mathrm{~K}$; (e) $551 \mathrm{~K}$; (f) $558 \mathrm{~K}$; (g) $565 \mathrm{~K}$; and (h) just below $T_{m}$. 

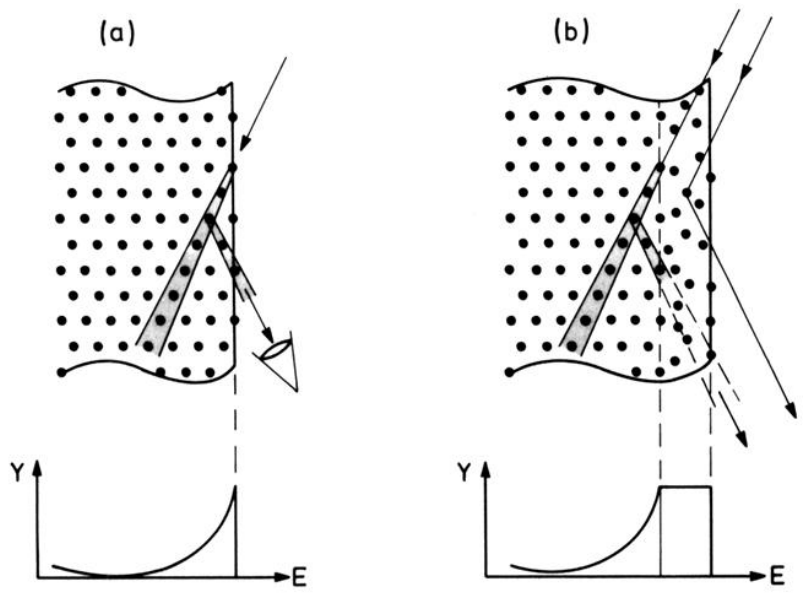

FIG. 2. Schematic representation of energy spectra for (a) a well-ordered crystal surface, and (b) a crystal covered by a disordered surface layer. Ion beam and detector are aligned with the [101] and [011] directions in the (1T1) crystal plane. Shadowing and blocking effects are indicated. 


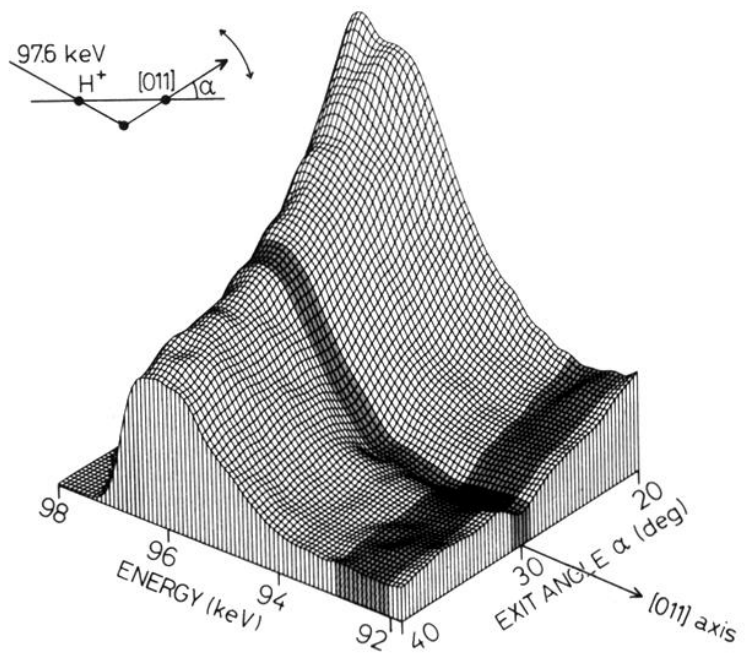

FIG. 5. Energy spectra measured at $600.5 \mathrm{~K}$ in a $20^{\circ}$ angular range around the [011] bulk axis. The shaded portions denote the $1.8^{\circ}$ angular window which is used to produce the energy spectra in Fig. 3, and the energy window, from 92 to $93 \mathrm{keV}$, which is used to produce the bulk blocking pattern in Fig. 6(a). The data have been smoothed for presentational purposes. The inset shows the scattering geometry (see also Fig. 2). 